\title{
Modelagem matemática do desenvolvimento foliar em mandioca ${ }^{1}$
}

\author{
Mathematical modeling of leaf development in the cassava
}

Flávia Kaufmann Samboranha², Nereu Augusto Streck ${ }^{3 *}$, Lilian Osmari Uhlmann e Luana Fernandes Gabriel²

\begin{abstract}
RESUMO - O objetivo deste trabalho foi simular a emissão do número de folhas com dois modelos matemáticos, um que considera o efeito linear e outro que considera o efeito não linear da temperatura do ar sobre o aparecimento de folhas em mandioca, e considerando duas versões de cada modelo, uma versão com a temperatura média diária do ar e outra com a temperatura mínima e máxima diária do ar, e validar o modelo de Wang; Engel para a mandioca. O modelo linear é o modelo do filocrono e o não linear é o modelo de Wang; Engel. Para estimar os coeficientes dos modelos foi usado um conjunto de dados de número de folhas na haste principal e na ramificação simpodial de primeira e segunda ordem da variedade de mandioca Fepagro RS 13, coletados em experimentos conduzidos durante os anos agrícolas 2005/2006 e 2007/2008. A avaliação dos modelos foi realizada com dados independentes coletados nos anos agrícolas de 2006/2007, 2008/2009, 2009/2010 e 2010/2011. O modelo Wang; Engel proporcionou melhor estimativa para o número de folhas em mandioca, com menor valor de raiz do quadrado médio do erro, comparado com o modelo do filocrono.
\end{abstract}

Palavras-chave: Manihot esculenta. Análise Foliar. Soma térmica.

\begin{abstract}
The object of this work was to simulate the total number of leaves issued, by using two mathematical models; one to consider the linear effect, and one the nonlinear effect of air temperature on leaf appearance in the cassava. Two versions of each model were used, one version for the mean daily air temperature and the other for the daily minimum and maximum air temperatures. The Wang-Engel model for the cassava was also validated. For the linear model, the phylochron model was used and for the nonlinear, that of Wang-Engel. To estimate the coefficients of the models, a data-set of the number of leaves on the main stem and on the first and second order sympodial branching of the cassava, variety Fepagro RS 13, was used, the data being collected in experiments conducted during the 2005/2006 and 2007/2008 growing seasons. Evaluation of the models was carried out using independent data collected in the 2006/2007, 2008/2009, 2009/2010 and 2010/2011 seasons. The Wang-Engel model gave a better estimate for the number of leaves in the cassava, with lower values for the root mean square error compared to the phylochron model.
\end{abstract}

Key words: Manihot esculenta. Number of leaves. Air temperature.

\footnotetext{
*Autor para correspondência

${ }^{1}$ Recebido para publicação em 11/05/2012; aprovado em 06/06/2013

Parte da Dissertação de Mestrado da primeira autora apresentada ao Programa de Pós-Graduação em Engenharia Agrícola, CCR/UFSM

${ }^{2}$ Programa de Pós-Graduação em Engenharia Agrícola, Universidade Federal de Santa Maria, Santa Maria-RS, Brasil, artemis.ufsm@ @otmail.com, luana2fernandes@gmail.com

${ }_{3}^{3}$ Departamento de Fitotecnia, CCR/UFSM, Santa Maria-RS, Brasil, nstreck2@yahoo.com.br

${ }^{4}$ Universidade Federal de Santa Maria, Santa Maria-RS, Brasil, liliuhlmann@ yahoo.com.br
} 


\section{INTRODUÇÃO}

A mandioca (Manihot esculenta Crantz) tem um papel importante na segurança alimentar das regiões tropicais, pois é um dos alimentos energéticos mais importantes nestas regiões (FOOD AND AGRICULTURE ORGANIZATION THE OF UNITED NATIONS, 2011). Além da alimentação humana, a mandioca também é utilizada na alimentação animal, onde tem grande importância nas pequenas propriedades rurais do Rio Grande do Sul (RS).

Modelos matemáticos são uma simplificação da realidade, que descrevem as interações existentes nos agroecossistemas. Uma alternativa ao método da soma térmica, que é um modelo linear, para descrever o efeito da temperatura do ar sobre o desenvolvimento vegetal, são os modelos multiplicativos que empregam funções de resposta não lineares para representar o efeito dos fatores abióticos sobre o desenvolvimento vegetal (STRECK et al., 2003a, 2003b), como o modelo de Wang e Engel (1998) (WE).

A emissão de folhas é um processo do desenvolvimento vegetal. Ao se integrar a taxa de emissão de folhas no tempo, tem-se o número de folhas acumulados (NF) em uma haste, considerada uma excelente medida de tempo vegetal (STRECK et al., 2003a). Portanto, o cálculo da emissão de folhas é um importante componente em modelos de simulação do desenvolvimento de culturas agrícolas (HODGES, 1991), incluindo a mandioca (MATTHEWS; HUNT, 1994).

$\mathrm{O}$ NF pode ser estimado por meio da taxa de aparecimento de folhas (TAF) através do filocrono, definido como o intervalo de tempo para o surgimento de folhas sucessivas em uma haste, o qual tem base na soma térmica (SCHONS et al., 2007). Outra forma de estimar a TAF é através de modelos como o WE. No modelo do filocrono pressupõe-se uma relação linear entre TAF e temperatura do ar (XUE; WEISS; BAEZING, 2004). No WE, a função de resposta da TAF à temperatura do ar [f(T)] é não linear, descrita por uma função beta (WANG; ENGEL, 1998). A $\mathrm{f}(\mathrm{T})$ é multiplicada pela taxa máxima de aparecimento de folhas em um dia, e por meio dessa multiplicação calculase a taxa atual de aparecimento das folhas.

A temperatura do ar é o principal fator abiótico que governa o aparecimento de folhas em mandioca (SCHONS et al., 2007). O modelo de WE tem melhor desempenho do que o método da soma térmica para descrever a emissão de folhas em culturas agrícolas, como melão (STRECK, 2002), kiwi (STRECK, 2003), trigo (XUE; WEISS; BAEZING, 2004), e arroz (STRECK; BOSCO; LAGO, 2008). O modelo de WE ainda não foi calibrado e testado em mandioca, o que constituiu em motivação para este estudo. Além disso, há duas maneiras de entrar com a temperatura para calcular a soma térmica diária (STd) no modelo do filocrono ou a $\mathrm{f}(\mathrm{T})$ diária no WE. A primeira abordagem calcula a STd ou a $\mathrm{f}(\mathrm{T})$ com a temperatura média diária do ar (MARTINS; STRECK, 2007) e a segunda calcula uma STd ou uma $\mathrm{f}(\mathrm{T})$ para cada valor de temperatura mínima e máxima diária e, após, calcula a média das STds ou $\mathrm{f}(\mathrm{T}) \mathrm{s}$ diárias (XUE; WEISS; BAEZING, 2004).

O objetivo deste trabalho foi simular a emissão do número de folhas (NF) com dois modelos matemáticos, um que considera o efeito linear e outro que considera o efeito não linear da temperatura do ar sobre o aparecimento de folhas em mandioca, considerando duas versões de cada modelo, uma versão com a temperatura média diária do ar (Tmed) e outra com a temperatura mínima e máxima diária do ar (Tmm), e validar o modelo de Wang; Engel para a mandioca.

\section{MATERIAL E MÉTODOS}

Os dados de NF da variedade de mandioca Fepagro - RS 13 foram coletados em experimentos de campo conduzidos no Departamento de Fitotecnia da Universidade Federal de Santa Maria (latitude: $29^{\circ} 43^{\text {' }}$ S; longitude: $53^{\circ} 43^{\prime} \mathrm{W}$; altitude: $95 \mathrm{~m}$ ), nos anos agrícolas 2005/2006, 2006/2007, 2008/2009, 2009/2010 e 2010/2011. O local de estudo é indicado para o plantio de mandioca no RS, pelo zoneamento agroclimático da cultura (MALUF; MATZENAUER; MALUF, 2011). O solo da área é uma transição entre a Unidade de Mapeamento São Pedro (Argissolo Vermelho distrófico arênico) e a Unidade de Mapeamento Santa Maria (Alissolo Hipocrômico argilúvico típico) (STRECK et al., 2008b), com teor de matéria orgânica próximo a $2 \%$. Segundo a classificação de Köppen, o clima deste local é do tipo subtropical úmido com verões quentes e sem estação seca definida (Cfa), com temperatura média anual de $18,8^{\circ} \mathrm{C}$ e precipitação pluviométrica total anual de $1.686,1 \mathrm{~mm}$.

O preparo do solo em todos os anos foi realizado de forma convencional e a adubação seguiu a recomendação técnica para a cultura da mandioca. Os dados diários de temperatura mínima (TN) e máxima (TX) do ar e de precipitação pluviométrica foram coletados na Estação Climatológica Principal do $8^{\circ}$ Distrito de Meteorologia do Instituto Nacional de Meteorologia (DISME/INMET), localizada no Departamento de Fitotecnia da Universidade Federal de Santa Maria, a aproximadamente $100 \mathrm{~m}$ da área experimental. 
A metodologia utilizada foi a mesma em todos os anos agrícolas, e consistiu em contar, uma vez por semana, o NF acumuladas na haste principal (HP), na ramificação simpodial de primeira ordem (RS1) e segunda ordem (RS2). O NF nas hastes da mandioca foi coletado em todos os experimentos com o critério de Schons et al. (2007), sendo uma folha considerada visível quando as bordas de um dos lóbulos desta folha não mais se tocarem.

Dois modelos foram utilizados para estimar a emissão de folhas em mandioca, o modelo do filocrono e o modelo de WE. A representação gráfica dos dois modelos de emissão de folhas está ilustrada na Figura 1. Em cada modelo foram usadas duas versões: uma versão com a temperatura média diária do ar (Tmed) e uma versão com a TN e TX diárias do ar (Tmm).

Figura 1 - Representação gráfica da resposta da emissão de folhas à temperatura do ar em mandioca no modelo linear (Filocrono) e no modelo não linear de Wang; Engel (WE). STd é a soma térmica diária em ${ }^{\circ} \mathrm{C}$ dia e $\mathrm{f}(\mathrm{T})$ é a função de temperatura do modelo WE

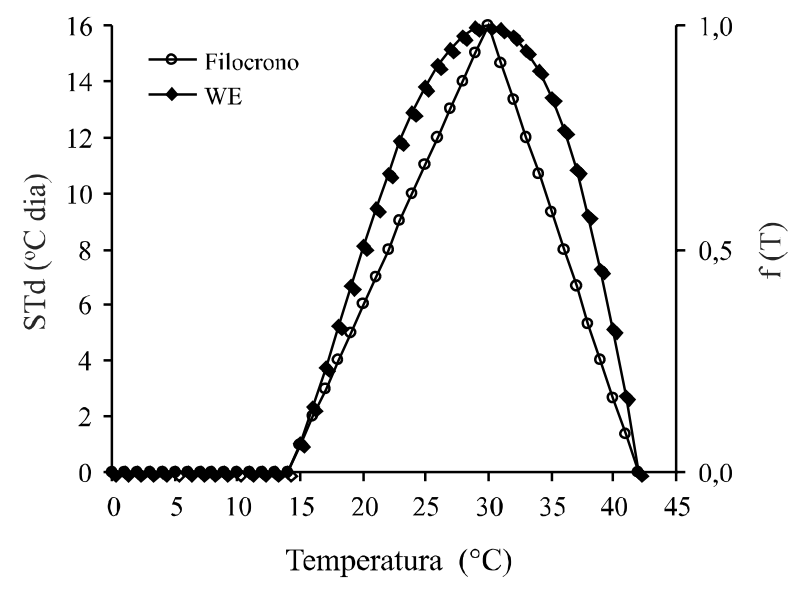

A soma térmica diária ( $\mathrm{STd},{ }^{\circ} \mathrm{C}$ dia $)$ foi calculada por (MATTHEWS; HUNT, 1994):

$\mathrm{STd}=(\mathrm{T}-\mathrm{Tb}) .1$ dia $\left\{{ }^{\circ} \mathrm{C}\right.$ dia $\}$

quando a $\mathrm{Tb}<\mathrm{T} \leq \mathrm{Tot} \mathrm{e}$,

$\mathrm{STd}=(\mathrm{Tot}-\mathrm{Tb}) \cdot(\mathrm{Tmax}-\mathrm{T}) /(\mathrm{Tmax}-\mathrm{Tot}) \cdot 1 \mathrm{dia}\left\{{ }^{\circ} \mathrm{C} \mathrm{dia}\right\}$

quando Tot $<\mathrm{T} \leq \mathrm{Tmax}$, em que $\mathrm{Tb}$, Tot e Tmax são as temperaturas cardinais para aparecimento de folhas em mandioca. $\mathrm{Tb}=14{ }^{\circ} \mathrm{C}$ (SCHONS et al., 2007), Tot $=30{ }^{\circ} \mathrm{C}$ e $\operatorname{Tmax}=42{ }^{\circ} \mathrm{C}$ (MATTHEWS; HUNT, 1994), e $\mathrm{T}$ temperatura diária do ar $\left({ }^{\circ} \mathrm{C}\right)$.
A soma térmica acumulada (STa) foi calculada por:

$\mathrm{STa}=\sum \mathrm{STd}\left\{{ }^{\circ} \mathrm{C}\right.$ dia $\}$

sendo $\sum \mathrm{STd}$, o somatório das somas térmicas diárias.

O filocrono $\left({ }^{\circ} \mathrm{C}\right.$ dia folha $\left.{ }^{-1}\right)$ foi estimado como sendo o inverso do coeficiente angular "a" da regressão linear (XUE; WEISS; BAEZING, 2004) entre o número de folhas nas hastes da planta de mandioca (NFHP, NFRS1 e NFRS2) e a STa a partir da data da emergência ou a partir das datas de surgimento das ramificações simpodiais na planta.

O NF foi calculado por (STRECK; BOSCO; LAGO, 2008):

$\mathrm{NF}=\mathrm{STa} / \mathrm{FILO}\{$ folhas $\}$

O modelo de WE tem a forma geral (WANG; ENGEL, 1998):

$\mathrm{TAF}=\mathrm{TAFmax} . \mathrm{f}(\mathrm{T})$

em que TAFmax é a taxa diária máxima de aparecimento de folhas (folhas $\operatorname{dia}^{-1}$ ) e f(T) é a função resposta à temperatura.

A função de temperatura $\mathrm{f}(\mathrm{T})$ é uma função beta que varia de zero a um. A f(T) é definida como (STRECK, 2002; 2003; WANG; ENGEL, 1998):

$\mathrm{f}(\mathrm{T})=\left[2(\mathrm{~T}-\mathrm{Tb})^{\alpha}(\mathrm{Tot}-\mathrm{Tb})^{\alpha}-(\mathrm{T}-\mathrm{Tb})^{2 \alpha}\right] /(\text { Tot-Tb })^{2 \alpha}$

quando $\mathrm{Tb} \leq \mathrm{T} \leq \mathrm{Tmax}$

$\mathrm{f}(\mathrm{T})=0$

quando $\mathrm{T}<\mathrm{Tb}$ ou $\mathrm{T}>\mathrm{T} \max$

$\alpha=\ln 2 / \ln [(\mathrm{Tmax}-\mathrm{Tb}) /(\mathrm{Tot}-\mathrm{Tb})]$

em que Tb, Tot e Tmax são as temperaturas cardinais para TAF e $\mathrm{T}$ é a temperatura média diária do ar. As temperaturas cardinais da mandioca na $\mathrm{f}(\mathrm{T})$ são as mesmas do filocrono.

Para calcular o NF no modelo WE, somam-se os valores diários de TAF, a partir da data de emergência ou das ramificações simpodiais (RS1 e RS2) da mandioca. O NF foi calculado por (WANG; ENGEL, 1998):

$\mathrm{NF}=\sum \mathrm{TAF}\{$ folhas $\}$

As duas versões do modelo do filocrono e de WE referem-se à maneira como a temperatura do ar foi usada no cálculo da STd nas equações (1) e (2), e na $f(T)$ nas equações (6) e (7): versão FILOTmed e WETmed, em que Tmed é a temperatura média diária do ar calculada pela média aritmética entre a TN e a TX diária do ar; versão FILOTmm e WETmm, calculase a uma STd e uma $\mathrm{f}(\mathrm{T})$ com a TN e outra com a TX, e depois calcula-se a média aritmética das duas STd e das duas $\mathrm{f}(\mathrm{T}) \mathrm{s}$ diárias. 
Os coeficientes FILO (equação 4) e TAFmax (equação 5) são dependentes do genótipo e foram estimados com dados de NF da HP, RS1 e RS2 da variedade Fepagro - RS 13, coletados nos experimentos dos anos agrícolas 2005/2006 e 2007/2008. Para determinar FILO calculou-se o filocrono para cada conjunto de dados dos anos agrícolas, e fez-se a média aritmética dos valores de filocrono obtidos para cada haste da planta nas versões Tmed e Tmm. Para determinar a TAFmax nas duas versões, utilizou-se o método dos mínimos quadrados. Fez-se a análise de variância e teste de Tukey a $5 \%$ de probabilidade para testar a diferença de FILO e TAFmax entre HP, RS1 e RS2 em cada versão dos modelos. Antes de realizar a análise de variância, os dados de FILO e TAFmax foram testados para normalidade usando-se o teste de Jarque-Bera a $5 \%$.

Para avaliar o desempenho dos modelos foram utilizados dados independentes de NF coletados em experimentos nos anos agrícolas 2006/2007, 2008/2009, 2009/2010 e 2010/2011. As estatísticas usadas para avaliar o desempenho dos modelos foram: Raiz do quadrado médio do erro (RQME) (JANSSEN; HEUBERGER, 1995), Índice BIAS (DE LEITE; ANDRADE, 2002), Índice de concordância (dw) (WILLMOTT, 1981), coeficiente de correlação (r); Índice de confiança(c) (DECAMARGO; SENTELHAS, 1997), erro sistemático (QMEs) e não sistemático (QMEns) (STRECK et al. 2008a; WILLMOTT ,1981) e a estatística $E_{12}$ (STRECK, 2002; STRECK et al., 2008c):

$\mathrm{RQME}=\left[\sum(\mathrm{Si}-\mathrm{Oi})^{2} / \mathrm{n}\right]^{0,5}$

em que Si são os valores simulados, Oi são os valores observados e $\mathrm{n}$ é o número de observações.

$\mathrm{BIAS}=\left(\sum \mathrm{Si}-\sum \mathrm{Oi}\right) / \sum \mathrm{Oi}$

$\mathrm{dw}=1-\left[\sum(\mathrm{Si}-\mathrm{Oi})^{2}\right] /\left[\sum(|\mathrm{Si}-\mathrm{O}|)+(|\mathrm{Oi}-\overline{\mathrm{O}}|)\right]^{2}$

$\mathrm{r}=\sum(\mathrm{Oi}-\overline{\mathrm{O}})(\mathrm{Si}-\mathrm{S}) /\left\{\left[\sum(\mathrm{Oi}-\overline{\mathrm{O}})^{2}\right]\left[\sum(\mathrm{Si}-\mathrm{S})^{2}\right]^{0,5}\right.$

em que Ō é a média dos valores observados e $\mathrm{S}$ é a média dos valores simulados.

$\mathrm{c}=\mathrm{rxdw}$

em que $r$ é o coeficiente de correlação e dw é o coeficiente de concordância.

QMEs $=\left[\sum(\hat{S} i-O i)^{2}\right] / n$

$\mathrm{QMEns}=\left[\sum(S i-\hat{S} i)^{2}\right] / n$

$\mathrm{E}_{12}=\mathrm{QME}_{1} / \mathrm{QME}_{2}$

em que QME $\mathrm{Q}_{1}$ Q $\mathrm{QE}_{2}$ são o quadrado médio do erro de simulações para o modelo 1 (WE) e modelo 2 (filocrono), respectivamente.
$\mathrm{QME}_{1}=\sum(\mathrm{S} 1 \mathrm{i}-\mathrm{Oi})^{2} / n$

$\mathrm{QME}_{2}=\sum(\mathrm{S} 2 i-\mathrm{Oi})^{2} / n$

sendo S1i os valores simulados para o modelo $1 \mathrm{e}$ S2i para o modelo 2 .

\section{RESULTADOS E DISCUSSÃO}

Nos seis anos agrícolas a precipitação pluviométrica variou desde anos abaixo da normal (2010/2011) até anos acima da normal (2009/2010) climatológica para Santa Maria, RS (Tabela 1). No ano agrícola de 2006/2007, o total pluviométrico precipitado foi de $1.034,3 \mathrm{~mm}$ com déficit calculado de $67,8 \mathrm{~mm}$, neste ano foi registrado o fenômeno El Niño com intensidade fraca e o maior volume mensal precipitado foi em outubro de 2006 (208,6 mm). O ano agrícola de 2007/2008 foi de La Ninã com intensidade moderada e precipitação abaixo da normal (anomalias negativas), exceto para o mês de dezembro de 2007 (68,8 mm). No ano agrícola 2009/2010 o volume acumulado de chuva foi $1.567,8 \mathrm{~mm}$ em decorrência do EL Niño de intensidade moderada, com chuvas acima da normal em novembro e dezembro de 2009 e janeiro de 2010. No ano agrícola de 2010/2011 a precipitação acumulada foi de apenas 637,8 mm, em função do fenômeno La Niña, com déficit de precipitação chegando a $200,8 \mathrm{~mm}$ para o período de outubro de 2010 a março de 2011 (Tabela 1).

De acordo com o teste de normalidade de JarqueBera, os valores dos coeficientes FILO e TAFmax nas versões $\mathrm{T}_{\text {med }}$ e $\mathrm{T}_{\mathrm{mm}}$ seguem uma distribuição normal. Assim, foi realizada uma ANOVA (Tabela 2) e os coeficientes FILO utilizados no modelo do filocrono e TAFmax utilizados no modelo de WE estão ilustrados na Tabela 3. Pela ANOVA (Tabela 2), há diferença no FILO e na TAFmax entre as hastes (HP, RS1 e RS2) nas duas versões dos modelos. O coeficiente FILO aumentou conforme surgem ramificações na planta, embora as diferenças entre HP e RS1 não sejam significativas (Tabela 3), indicando um maior intervalo de tempo entre a emissão de duas folhas sucessivas nas hastes da RS2 do que em RS1 e HP. Fagundes et al. (2009) sugerem que o aumento do filocrono nas hastes das ramificações simpodiais é devido ao maior aporte de nutrientes e água dentro da planta, e a cada nova ramificação novas hastes surgem necessitando de mais água e nutrientes. $\mathrm{O}$ coeficiente TAFmax, que representa a velocidade de aparecimento de folhas em um dia, foi maior na HP e na RS1 e menor na RS2, confirmando a menor velocidade de aparecimento de folhas na ramificação simpodial de segunda ordem (Tabela 3 ).

Os valores de número de folhas (NF) observados e simulados pelos modelos do filocrono e de WE, nas 
Tabela 1 - Valores médios mensais de temperatura mínima, média e máxima do ar, radiação solar global e precipitação mensal acumulada durante o período de emissão de folhas na variedade de mandioca Fepagro - RS 13 nos anos agrícolas 2005/2006 a 2010/2011, em Santa Maria, RS

\begin{tabular}{|c|c|c|c|c|c|c|}
\hline \multirow{3}{*}{ Ano Agrícola } & \multirow{3}{*}{ Mês } & \multicolumn{3}{|c|}{ Temperatura } & \multirow{3}{*}{ Radiação Solar Global } & \multirow{3}{*}{ Precipitação Acumulada } \\
\hline & & Mín. & Méd. & Máx. & & \\
\hline & & $\left({ }^{\circ} \mathrm{C}\right)$ & $\left(\mathrm{MJ} \mathrm{m}^{-2} \mathrm{dia}^{-1}\right)$ & $(\mathrm{mm})$ & & \\
\hline \multirow{8}{*}{$2005 / 2006$} & Outubro & 14,5 & 18,8 & 23,1 & 13,0 & $284,0(+139,0)^{*}$ \\
\hline & Novembro & 15,7 & 22,6 & 29,4 & 19,4 & $57,2(-75,0)$ \\
\hline & Dezembro & 17,3 & 23,8 & 30,3 & 21,3 & $101,2(-31,6)$ \\
\hline & Janeiro & 20,7 & 26,3 & 31,9 & 19,2 & $197,7(+52,6)$ \\
\hline & Fevereiro & 19,0 & 25,1 & 31,2 & 19,1 & $45,5(-84,7)$ \\
\hline & Março & 18,1 & 24,2 & 30,3 & 16,2 & $80,2(-71,5)$ \\
\hline & Abril & 14,2 & 20,1 & 26,0 & 13,0 & $68,6(-66,1)$ \\
\hline & Média & 17,1 & 23,0 & 28,9 & 17,3 & Total $834,4(-138,2)$ \\
\hline \multirow{9}{*}{$2006 / 2007$} & Outubro & 15,6 & 21,2 & 26,9 & 16,5 & $208,6(+63,0)$ \\
\hline & Novembro & 16,5 & 21,8 & 27,1 & 18,0 & $134,6(+2,4)$ \\
\hline & Dezembro & 19,6 & 25,9 & 32,2 & 20,3 & $84,2(-49,3)$ \\
\hline & Janeiro & 20,5 & 25,7 & 30,8 & 18,9 & $163,9(+18,8)$ \\
\hline & Fevereiro & 19,5 & 25,1 & 30,8 & 18,8 & $145,2(+15,0)$ \\
\hline & Março & 20,4 & 25,3 & 30,1 & 13,7 & $173,6(+21,9)$ \\
\hline & Abril & 16,7 & 22,2 & 27,7 & 12,2 & $21,4(-113,3)$ \\
\hline & Maio & 9,3 & 14,8 & 20,1 & 9,7 & $102,8(-26,3)$ \\
\hline & Média & 17,3 & 22,7 & 28,2 & 16,0 & Total 1034,3 $(-67,8)$ \\
\hline \multirow{7}{*}{$2007 / 2008$} & Outubro & 16,6 & 21,4 & 26,0 & 12,4 & $113,2(-37,2)$ \\
\hline & Novembro & 14,6 & 20,9 & 27,1 & 18,9 & $105,0(-27,2)$ \\
\hline & Dezembro & 18,3 & 24,8 & 31,3 & 20,4 & $202,1(+68,8)$ \\
\hline & Janeiro & 19,1 & 24,8 & 30,4 & 20,6 & $95,7(-49,4)$ \\
\hline & Fevereiro & 18,7 & 24,7 & 30,6 & 18,0 & $127,3(-2,9)$ \\
\hline & Março & 18,1 & 23,6 & 29,1 & 16,4 & $106,9(-44,8)$ \\
\hline & Média & 17,6 & 23,3 & 29,1 & 17,8 & Total 750,3 $(-88,3)$ \\
\hline \multirow{5}{*}{ 2008/2009 } & Outubro & 15,0 & 19,8 & 24,6 & 14,6 & $255,3(+109,4)$ \\
\hline & Novembro & 17,2 & 23,2 & 29,2 & 20,3 & $43,9(-88,3)$ \\
\hline & Dezembro & 18,1 & 24,5 & 30,8 & 21,1 & $31,7(-101,8)$ \\
\hline & Janeiro & 18,1 & 24,0 & 29,9 & 19,7 & $162,1(+17,0)$ \\
\hline & Média & 17,1 & 22,9 & 28,6 & 18,9 & Total 493,0 $(-63,7)$ \\
\hline \multirow{8}{*}{ 2009/2010 } & Outubro & 13,5 & 19,5 & 25,5 & 16,7 & $108,7(-37,2)$ \\
\hline & Novembro & 19,2 & 23,8 & 28,3 & 13,6 & $480,9(+348,7)$ \\
\hline & Dezembro & 19,9 & 24,9 & 29,8 & 19,3 & $305,7(+172,2)$ \\
\hline & Janeiro & 20,6 & 25,4 & 30,1 & 17,8 & $405,9(+260,8)$ \\
\hline & Fevereiro & 22,0 & 26,9 & 31,8 & 16,9 & $124,7(-5,5)$ \\
\hline & Março & 19,2 & 24,6 & 30,1 & 15,7 & $25,1(-126,6)$ \\
\hline & Abril & 14,2 & 20,1 & 26,1 & 12,3 & $116,8(-17,9)$ \\
\hline & Média & 18,3 & 23,6 & 28,8 & 16,1 & Total $1567,8(+594,5)$ \\
\hline
\end{tabular}


Continuação Tabela 1

\begin{tabular}{lllllcc}
\hline & Outubro & 12,7 & 18,6 & 24,4 & 16,8 & $49,3(-96,6)$ \\
& Novembro & 14,9 & 21,3 & 27,7 & 19,1 & $83,0(-49,2)$ \\
\multirow{2}{*}{$2010 / 2011$} & Dezembro & 18,4 & 24,4 & 30,4 & 21,2 & $157,9(+24,4)$ \\
& Janeiro & 21,8 & 27,1 & 32,5 & 19,5 & $127,1(-18,0)$ \\
& Fevereiro & 20,9 & 25,4 & 30,0 & 17,3 & $165,6(+35,4)$ \\
& Março & 18,2 & 23,5 & 28,9 & 16,3 & $54,9(-96,8)$ \\
\cline { 2 - 6 } & Média & 17,8 & 23,4 & 29,0 & 18,3 & Total 637,8 (-200,8) \\
\hline
\end{tabular}

*Valores entre parêntesis representam a anomalia de precipitação (valor registrado - valor normal)

Tabela 2 - Quadro de análise da variância (ANOVA) para as variáveis FILO - versão $\mathrm{T}_{\text {med }}$, FILO - versão $\mathrm{T}_{\mathrm{mm}}$, TAFmax _versão $\mathrm{T}_{\mathrm{med}} \mathrm{e}$ TAFmax _ versão $\mathrm{T}_{\mathrm{mm}}$. GL= Graus de Liberdade, $\mathrm{SQ}=$ Soma de Quadrados, $\mathrm{QM}=$ Quadrado médio

\begin{tabular}{|c|c|c|c|c|c|}
\hline Fonte de variação & GL & SQ & $\mathrm{QM}$ & Valor-F & Prob $>F$ \\
\hline \multicolumn{6}{|c|}{ Variável FILO - Versão $\mathrm{T}_{\text {med }}$} \\
\hline Modelo & 4 & 62,0161 & 15,5040 & \multirow{2}{*}{27,21} & \multirow{2}{*}{0,0108} \\
\hline Resíduo & 3 & 1,7091 & 0,5697 & & \\
\hline \multicolumn{6}{|c|}{ Variável FILO - Versão $\mathrm{T}_{\mathrm{mm}}$} \\
\hline Modelo & 4 & 55,8518 & 13,9629 & \multirow{2}{*}{41,65} & \multirow{2}{*}{0,0058} \\
\hline Resíduo & 3 & 1,0058 & 0,3353 & & \\
\hline \multicolumn{6}{|c|}{ Variável TAFmax - Versão $\mathrm{T}_{\text {med }}$} \\
\hline Modelo & 4 & 0,1153 & 0,02883 & \multirow{2}{*}{65,56} & \multirow{2}{*}{0,0030} \\
\hline Resíduo & 3 & 0,0013 & 0,00044 & & \\
\hline \multicolumn{6}{|c|}{ Variável TAFmax - Versão $\mathrm{T}_{\mathrm{mm}}$} \\
\hline Modelo & 4 & 0,1649 & 0,04123 & \multirow{2}{*}{79,15} & \multirow{2}{*}{0,0023} \\
\hline Resíduo & 3 & 0,0016 & 0,0005 & & \\
\hline
\end{tabular}

Tabela 3 - Coeficientes FILO (filocrono no modelo linear) e TAFmax (taxa máxima de aparecimento de folhas no modelo não linear) para a emissão de folhas em diferentes hastes da variedade de mandioca Fepagro - RS 13, em duas versões de temperatura, em Santa Maria, RS

\begin{tabular}{|c|c|c|c|}
\hline \multirow{2}{*}{ Versão ${ }^{(2)}$} & \multicolumn{3}{|c|}{ Haste $^{(1)}$} \\
\hline & HP & RS1 & RS2 \\
\hline \multicolumn{4}{|c|}{ Coeficiente FILO $\left({ }^{\circ} \mathrm{C}\right.$ dia folha $\left.{ }^{-1}\right)$} \\
\hline Tmed & $15,07 \mathrm{~b}$ & $16,76 \mathrm{~b}$ & $21,82 \mathrm{a}$ \\
\hline Tmm & $12,42 \mathrm{~b}$ & $14,22 \mathrm{~b}$ & $18,94 \mathrm{a}$ \\
\hline \multicolumn{4}{|c|}{ Coeficiente TAFmax (folhas dia $\left.{ }^{-1}\right)$} \\
\hline Tmed & $0,81 \mathrm{a}$ & $0,82 \mathrm{a}$ & $0,54 \mathrm{~b}$ \\
\hline Tmm & $0,95 \mathrm{a}$ & $0,98 \mathrm{a}$ & $0,64 \mathrm{~b}$ \\
\hline
\end{tabular}

${ }^{(1)} \mathrm{HP}=$ Haste principal; RS1= Primeira ramificação simpodial; $\mathrm{RS} 2=$ Segunda ramificação simpodial. Médias seguidas pela mesma letra na horizontal não diferem entre si pelo teste Tukey a 5; ${ }^{(2)} \mathrm{Tmed}=$ versão que usa a temperatura média diária do ar; Tmm= versão que usa a temperatura mínima e máxima diária do ar duas versões, são apresentados na Figura 2. A partir do valor do resíduo (valor simulado menos observado) apresentado nas inserções da Figura 2, constata-se uma superestimação do NF de, aproximadamente, quatro a cinco folhas com os dois modelos. A superestimação ocorreu mais no começo das observações quando as hastes possuem menos de 10 folhas (Figura 2). À medida que aumentou o NF, os resíduos se distribuíram mais em torno de zero, com uma tendência triangular mais aparente nas duas versões do modelo WE (Figura $2 b$ e $2 d$ ), indicando que neste modelo os resíduos tem algum viés.

Os valores das estatísticas indicam melhor desempenho do modelo de WE para simular o NF em mandioca. O RQME (Raiz do Quadrado Médio do Erro) foi menor com o modelo de WE em ambas versões (3,2 e 3,5 folhas), comparando com o modelo do filocrono (3,7 e 4,4 folhas) (Figura 2a, b, c, d). O componente sistemático (QMEs) foi menor para o modelo de WE nas versões Tmed e Tmm, confirmando 
Figura 2 - Número de folhas (NF) observado versus simulado na haste principal (HP), na primeira e segunda ramificação simpodial (RS1 e RS2) da variedade de mandioca Fepagro - RS 13, pelos modelos do filocrono e de Wang; Engel (WE) com duas versões de temperatura do ar (Tmed: a, b; Tmm: c, d) para os anos agrícolas 2006/2007 a 2010/2011. A linha cheia é a linha 1:1. O resíduo (NF simulado menos observado) versus o NF observado, e as estatísticas Raiz Quadrada Médio do Erro (RQME), índice BIAS, índice de concordância (dw), coeficiente de correlação (r) e índice de confiança (c) são apresentados nas inserções de cada painel. Santa Maria, RS, 2012
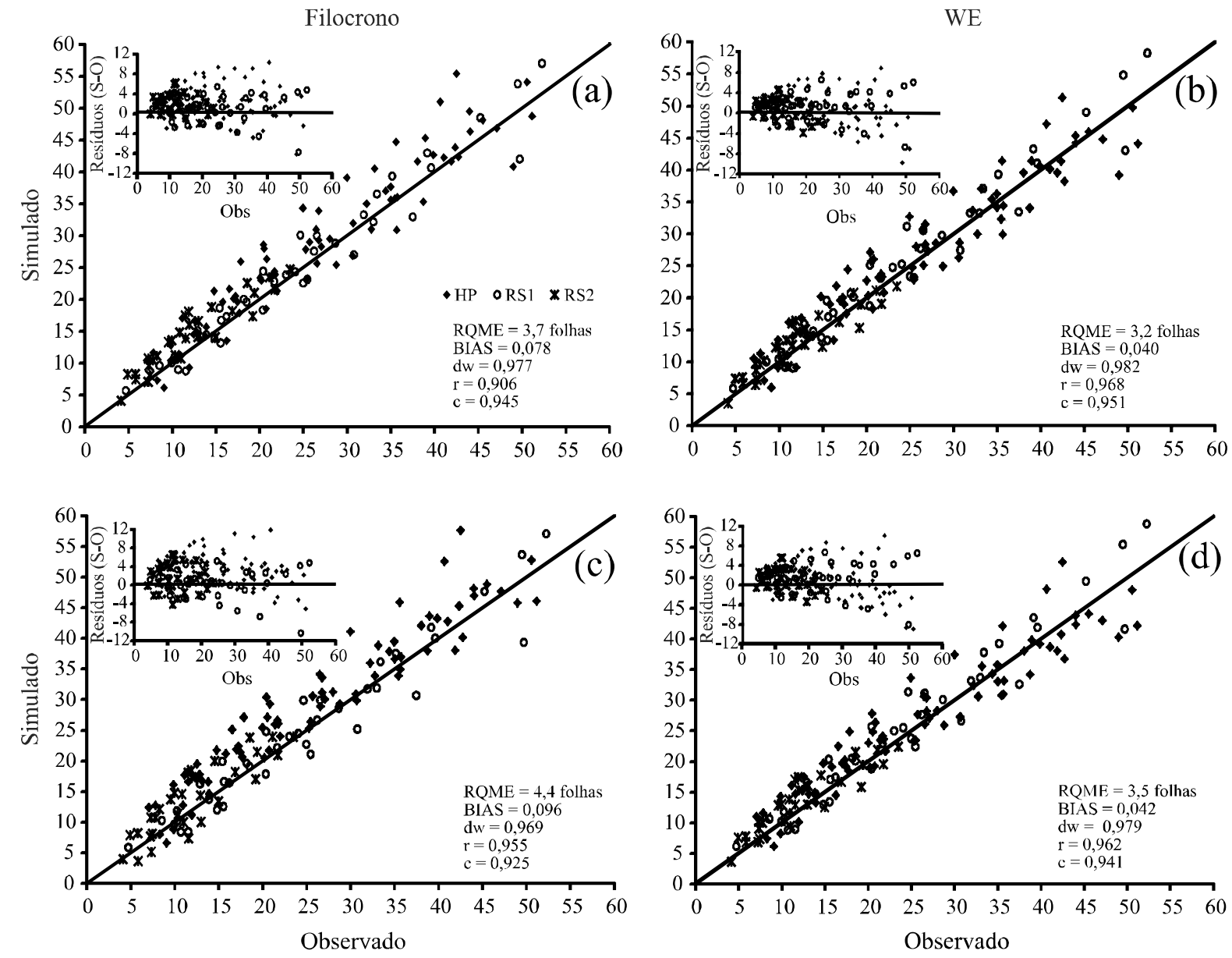

o melhor desempenho do modelo de WE. O QMEs foi menor para o modelo de WE nas versões Tmed (10,6\%) e $\operatorname{Tmm}(23,1 \%)$, em relação ao modelo do filocrono, com $23,1 \%$ na versão Tmed e $25,2 \%$ na versão Tmm. Já para o QMEns os menores valores foram no modelo do filocrono $(76,9 \%$ para a versão Tmed e $74,8 \%$ para a versão Tmm) comparado ao modelo WE (89,4\% para a versão Tmed e 76,9\% para a versão Tmm). Menor QMEs com o modelo de WE, também foi obtido por Streck et al. (2008a) e Xue, Weiss e Baezinger (2004) para a previsão de Estágio Haun em arroz (no Rio Grande do Sul, Brasil) e em trigo de inverno (em Nebraska, Estados Unidos), respectivamente.

Os valores positivos do índice BIAS indicam que os modelos superestimam o NF em todas as situações analisadas (Figura 2), mas os valores de BIAS foram

menores com o modelo WE nas duas versões (Figura 2b e 2d). De acordo com Schneider (1998), quanto mais próximo de zero for o valor de BIAS, menor é a tendência do modelo em estudo. A estatística BIAS junto com as demais estatísticas confirmam o desempenho superior do modelo de WE em relação ao filocrono. Pelo índice BIAS, a versão Tmed foi melhor que a Tmm, para os dois modelos (Figura 2). Os valores dos índices estatísticos coeficiente de determinação, dw $(0,979$ e 0,982$)$, coeficiente de correlação, r $(0,962$ e 0,968$)$ e índice de confiança, c $(0,941$ e 0,951$)$ foram elevados para o modelo de WE (Figura $2 \mathrm{~b}$ e 2d), indicando maior concordância e maior grau de associação (adesão) entre simulado e observado, que o modelo do filocrono, respectivamente. A estatística c indicou ótimo desempenho para o modelo de WE, considerando os critérios estabelecidos por De Camargo e Sentelhas (1997). 
A estatística $E_{12}$ indica a precisão relativa dos modelos e das versões de temperatura. Valores de $E_{12}$ menores que 1 indicam a superioridade de WEem relação ao filocrono dentro de cada versão. Para as versões, os valores de $\mathrm{E}_{12}$ abaixo de 1 indicam superioridade da versão Tmm em relação a Tmed dentro de cada modelo (STRECK et al., 2008c). A estatística $\mathrm{E}_{12}$ foi abaixo de 1 (0,758 na Tmed e 0,713 na Tmm), indicando que o modelo de WE teve melhor desempenho. Considerando as duas versões dos modelos, a estatística $\mathrm{E}_{12}$ indicou que a versão Tmed (1,040 no filocrono e 1,319 no WE) foi melhor.
Um destaque para o desempenho do modelo de WE e de ambas versões na estimativa do NF da HP no ano agrícola 2009/2010 está na Figura 3. Este ano agrícola foi de muitos dias nublados e chuvosos, nos meses de novembro e dezembro de 2009 e janeiro de 2010, devido ao fenômeno El Niño (Tabela 1). Houve grande superestimação do NF pelo modelo de WE na HP (Figura 3a e 3b). A hipótese para o fraco desempenho do modelo na HPe RS1 é de que a alta precipitação e muitos dias nublados reduziram a disponibilidade de radiação solar, o que pode ter causado redução na fotossíntese a ponto de afetar a velocidade de diferenciação de

Figura 3 - Evolução do número de folhas observado versus simulado pelo modelo de Wang;Engel (WE) com duas versões de temperatura do ar (Tmed: a, c, e; Tmm: b, d, f) na haste principal (HP), ramificação simpodial de primeira (RS1) e segunda ordem (RS2) da variedade de mandioca Fepagro - RS 13 para o ano agrícola 2009/2010. Santa Maria, RS, 2012. DAE= Dias após a emergência; DARS1= Dias após a ramificação simpodial de primeira ordem; DARS2= Dias após a ramificação simpodial de segunda ordem
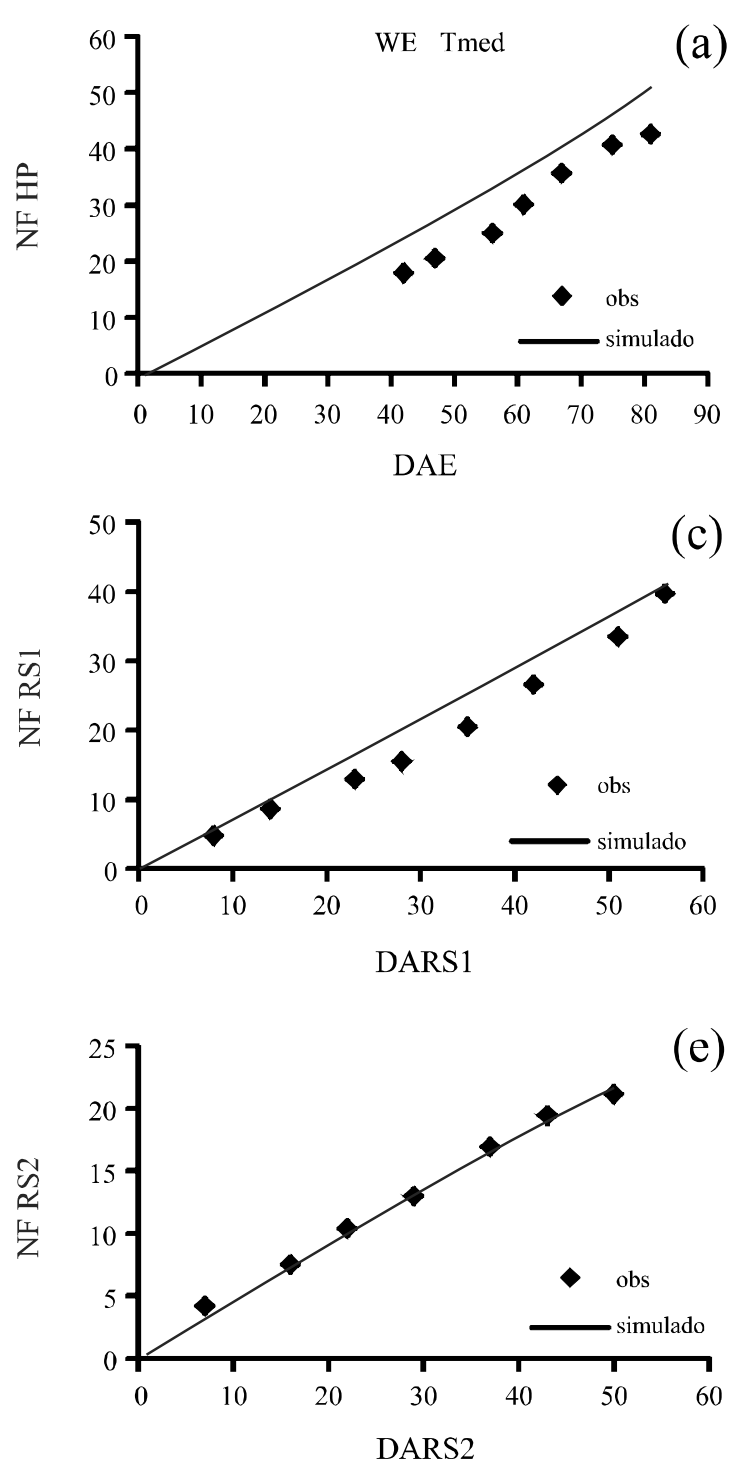

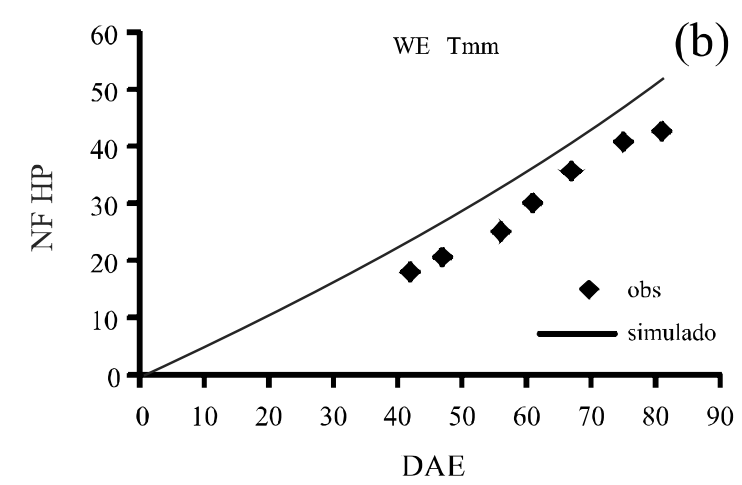

(d)
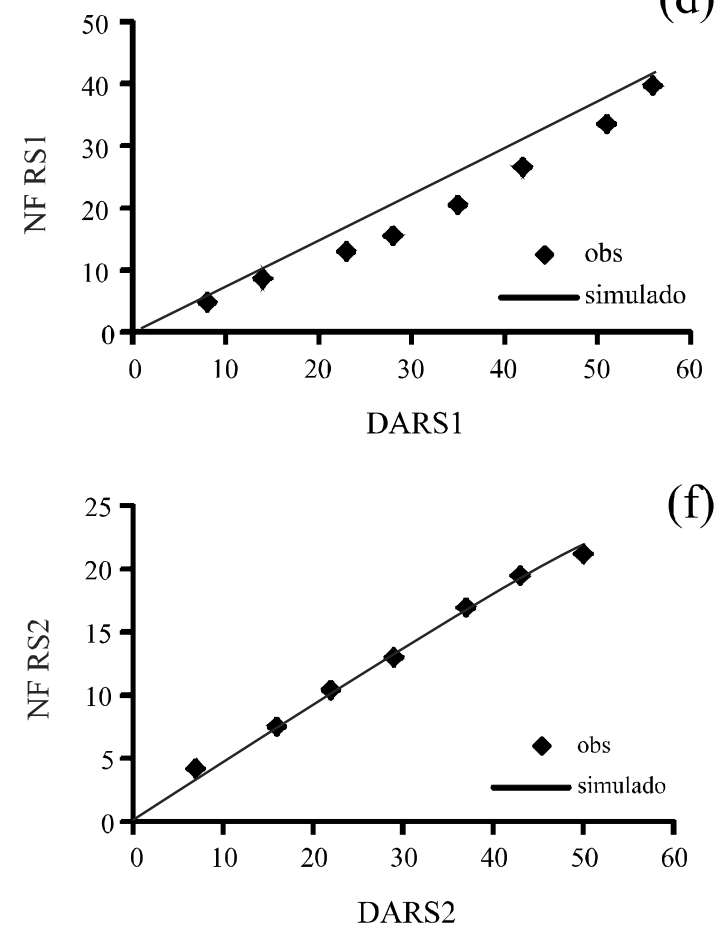
folhas no ápice meristemático (ALVES, 2006). Neste mesmo ano agrícola, as simulações foram boas para a RS1 (Figura 3c e 3d) e excelentes para a RS2 (Figura 3e e 3f). Em fevereiro, março e abril, quando ocorreu a emissão de folhas na RS1 e RS2, não houve excesso de precipitação (Tabela 1) e assim, provavelmente, a radiação solar não foi limitante para a emissão de folhas. Ressalta-se, a excelente simulação do número de folhas no final do período da emissão de folhas na RS2 (Figura 3e e 3f), quando, nos últimos quatro dias até o último valor observado (4 a 7 de abril de 2010), ocorreram temperaturas mínimas e máximas diárias do ar variando de 10,8 a $17,6^{\circ} \mathrm{C}$ e 21,0 a $24,2^{\circ} \mathrm{C}$, respectivamente, que são temperaturas baixas para emissão de folhas em mandioca, indicando que o modelo de WE capturou a baixa taxa de aparecimento de folhas.

A melhor performance do modelo WE é atribuída à resposta não linear do aparecimento de folhas em função da temperatura do ar (XUE; WEISS; BAEZING, 2004), a qual tem significado biológico, pois processos vegetais são resultados de mecanismos básicos enzimáticos que respondem à temperatura de forma não linear (WANG; ENGEL, 1998), e definição operacional dos coeficientes TAFmax e as temperaturas cardinais (Tb, Tot e Tmax) na $\mathrm{f}(\mathrm{T})$ (STRECK et al., 2008c). No modelo do filocrono, a resposta do aparecimento de folhas à temperatura é linear, o que não é totalmente aceitável do ponto de vista biológico, pois quando está próxima das temperaturas cardinais ( $\mathrm{Tb}$, Tot e Tmax), a resposta da TAF à temperatura é não linear (STRECK, 2002; XUE; WEISS; BAEZING, 2004).

É importante ressaltar que outras funções não lineares podem ser usadas para descrever a resposta do desenvolvimento das plantas àtemperatura, porém, algumas destas funções têm a desvantagem de serem compostas por coeficientes empíricos, como na função exponencial dupla (STRECK, 2004a), que não tem significado biológico. Segundo Streck (2004a, 2004b), tais desvantagens devem ser evitadas em estudos de modelagem, pois abordagens empíricas resultam em coeficientes que não podem ser usados em diferentes condições ambientais. $\mathrm{O}$ modelo de WE não tem esta desvantagem, e ainda, combina os efeitos do ambiente sobre a TAF de forma multiplicativa, o que é biologicamente aceito, uma vez que as interações entre ambiente e desenvolvimento vegetal são geralmente multiplicativas (STRECK et al., 2003a, 2003b).

Dentre as duas versões, observou-se que, as estatísticas descritivas indicaram a Tmed como superior a Tmm para estimar a TAF em mandioca, nos dois modelos. A hipótese para explicar o desempenho das versões pode estar na época de cultivo da mandioca. A planta é perene e nativa de ambiente tropical, e em condições subtropicais o cultivo é sazonal e durante a estação de cultivo, a temperatura do ar, em geral, situa- se na faixa intermediária entre a temperatura base e a ótima (Tabela 1), faixa em que a resposta da planta à temperatura é linear (STRECK, 2004 a,b; XUE; WEISS; BAEZING, 2004), e nestas condições os resultados de ambas versões do modelo de WE funcionam bem, pois a diferença de RQME entre as versões, no modelo de $\mathrm{WE}$, foi pequena ( $<0,5$ folhas) (Figura $2 \mathrm{~b}$ e $2 \mathrm{~d}$ ). No entanto, em regiões mais quentes, como as regiões Norte e Nordeste do Brasil ou em cenários climáticos futuros (INTERGOVERNMENTAL PANEL ON CLIMAGE CHANGE, 2007), em que a temperatura do ar é esperada ser acima da atual, espera-se que a planta de mandioca seja exposta à temperaturas do ar na faixa não linear de resposta (Figura 1) com mais freqüência. Também, em estudos utilizando cenários de mudança climática, se espera aumento, tanto de TMIN quanto de TMAX (WEISS; HAYS; WON, 2003) e, em alguns locais esse aumento pode ser assimétrico, com maiores aumentos na TMIN do que na TMAX, incluindo o Sul do Brasil (MARENGO; CAMARGO, 2008). Portanto, nestas regiões e condições futuras, indica-se usar a versão Tmm do modelo de WE para simular a emissão de folhas em plantas de mandioca.

\section{CONCLUSÕES}

1.Um modelo matemático não linear (modelo WE) é preferível para simular o desenvolvimento foliar em mandioca comparado a um modelo linear (filocrono);

2. Sugere-se usar a versão com a temperatura mínima e máxima (Tmm) do modelo WE especialmente se o modelo for usado em regiões mais quentes, como as regiões Norte e Nordeste do Brasil ou em cenários de Aquecimento Global.

\section{REFERÊNCIAS}

ALVES, A. A. C. Fisiologia da mandioca. In: EMBRAPA MANDIOCA E FRUTICULTURA TROPICAL. Aspectos socioeconômicos e agronômicos da mandioca. Cruz das Almas, BA; EMBRAPA, 2006. Cap. 7, p. 138-169.

DE CAMARGO, A. P. de; SEnTElhas, P. C. Avaliação do desempenho de diferentes métodos de estimativa da evapotranspiração potencial no Estado de São Paulo, Brasil. Revista Brasileira de Agrometeorologia, v. 5, n. 1, p. 89-97, 1997.

DE LEITE, H. G.; ANDRADE, V. C. L. de. Um método para condução de inventários florestais sem o uso de equações volumétricas. Revista Árvore, v. 26, n. 3, p. 321-328, 2002.

FAGUNDES, L. K. et al. Desenvolvimento vegetativo em diferentes hastes da planta de mandioca em função da época de plantio. Ciência Rural, v. 39, n. 3, p. 657-663, 2009. 
FOOD AND AGRICULTURE ORGANIZATION OF THE UNITED NATIONS. Production, crops. Disponível em: <http:// faostat.fao.org/site/339/default.aspx>. Acesso em: 30 mar. 2011.

HODGES, T. Predicting crop phenology. CRC, 1991. 233 p.

INTERGOVERNMENTAL PANEL ON CLIMATE CHANGE. Climate Change 2007: The Physical Science Basis. Contribution of Working Group I to the Fourth Assessment Report of the Intergovernmental Panel on Climate Change. New York: Cambridge University Press, 2007, 996 p.

JANSSEN, P. H. M.; HEUBERGER, P. S. C. Calibration of process-oriented models. Ecological Modelling, v. 83, n. 1/2, p. 55-56, 1995.

MARENGO, J. A.; CAMARGO, C. C. Surface air temperature trends in Southern Brazil for 1960-2002. International Journal of Climatology, v. 28, n. 7, p. 893-904, 2008.

MARTINS, F. B.; STRECK, N. A. Aparecimento de folhas em mudas de eucalipto estimado por dois modelos. Pesquisa Agropecuária Brasileira, v. 42, n. 8, p. 1091-1100, 2007.

MATTHEWS, R. B.; HUNT, L. A. GUMCAS: a model describing the growth of cassava (Manihot esculenta L. Crantz). Field Crops Research, v. 36, n. 1, p. 69-84, 1994.

MALUF, J. R. T.; MATZENAUER, R.; MALUF, D. E. Zoneamento Agroclimático da Mandioca no Estado do Rio Grande do Sul - Uma alternativa para a produção de etanol. Porto Alegre: FEPAGRO, 2011. (Boletim Fepagro, 22). 60 p.

SCHNEIDER, P. R. Análise de regressão aplicada à engenharia florestal. 2. ed. Santa Maria: Ed. UFSM/CEPEF, 1998. $236 \mathrm{p}$.

SCHONS, A. et al. Emissão de folhas e início da acumulação de amido em raízes de uma variedade de mandioca em função da época de plantio. Ciência Rural, v. 37, n. 6, p. 1586-1592, 2007.

STRECK, N. A. A generalized nonlinear air temperature response function for node appearance rate in muskmelon (Cucumis melo L.). Revista Brasileira de Agrometeorologia, v. 10, n. 1, p. 105-111, 2002.
STRECK, N. A. A generalized nonlinear temperature response function for some growth and developmantal parameters in kiwifruit (Actinidia deliciosa (A. Chev.) C. F. Liang \& A. R. Ferguson). Ciência Rural, v. 33, n. 2, p. 255-262, 2003.

STRECK, N. A. A temperature response function for development of the crysanthemum (Chrysanthemum $x$ morifolium Ramat.). Ciência Rural, v. 34, n. 1, p. 49-54, 2004a.

STRECK, N. A. A temperature response function for modeling leaf growth and development of the African violet (Saintpaulia ionantha Wendl.). Ciência Rural, v. 34, n. 1, p. 55-62, 2004 b.

STRECK, N. A. et al. Improving predictions of developmental stages in winter wheat: a modified Wang and Engel model. Agricultural and Forest Meteorology, v. 115, n. 3/4, p. 139-150, 2003a.

STRECK, N. A. et al. Incorporating a chronology response function into the prediction of leaf appearance rate in winter wheat. Annals of Botany, v. 92, n. 2, p. 181-190, 2003 b.

STRECK, N. A.; BOSCO, L. C.; LAGO, I. Simulating leaf appearance in rice. Agronomy Journal, v. 100, n. 03, p. 490-501, 2008.

STRECK, E. V. et al. Solos do Rio Grande do Sul. Porto Alegre: EMATER/RS; UFRGS, 2008b. 126 p.

STRECK, N. A. et al. Simulating maize phenology as a function of air temperature with a linear and a non linear model. Pesquisa Agropecuária Brasileira, v. 43, n. 4, p. 449-455, 2008 c.

WANG, E.; ENGEL, T. Simulation of phenological development of wheat crops. Agricultural Systems, v. 58, n. 1, p. 01-24, 1998.

WEISS, A.; HAYS, C.J.; WON, J. Assessing winter wheat response to climate change scenarios: a simulation study in the U.S. Great Plains. Climatic Change, v. 58, n. 1/2, p. 119-147, 2003.

WILlmotT, C. J. On the validation of models. Physical Geograph, v. 2, n. 1, p. 184-194, 1981.

XUE, Q.; WEISS, A.; BAENZIGER, P. S. Predicting leaf appearance in field-grown winter wheat: evaluating linear and non-linear models. Ecological Modelling, v. 175, n. 3, p. 261270, 2004. 